\title{
Home monitoring service was more effective than usual care in patients with essential hypertension
}

\author{
Rogers MA, Small D, Buchan DA, et al. Home monitoring service improves mean arterial pressure in patients with \\ essential hypertension. A randomized, controlled trial. Ann Intern Med 2001 Jun 5;134:1024-32.

\section{QUESTION: In patients with essential hypertension, does a home monitoring service (HMS) improve mean arterial pressure more than usual care?}

Design

Randomised (allocation concealed*), unblinded,* controlled trial with a median 11 weeks of follow up.

\section{Setting}

University affiliated primary care outpatient clinics in New York, USA.

\section{Patients}

121 patients (mean age $62 \mathrm{y}, 50 \%$ women) with essential hypertension who needed to change their antihypertensive treatment because of elevated blood pressure (BP) despite current use of antihypertensive medication, with undesirable side effects from the current antihypertensive drug, or with an office systolic $\mathrm{BP} \geqslant 180 \mathrm{~mm} \mathrm{Hg}$ or a diastolic $\mathrm{BP} \geqslant 110 \mathrm{~mm} \mathrm{Hg}$ with no current use of medication. In patients with diabetes mellitus, heart disease, stroke, nephropathy, peripheral arterial disease, or hypertensive retinopathy, an office systolic BP $\geqslant 130 \mathrm{~mm} \mathrm{Hg}$ or an office diastolic $\mathrm{BP} \geqslant 85 \mathrm{~mm} \mathrm{Hg}$ was needed to be eligible. Exclusion criteria included age $<18$ years, pregnancy, secondary hypertension, and lack of mental or physical capability to monitor BP at home. $92 \%$ completed the trial.

\section{Intervention}

60 patients were allocated to HMS, during which automatic BP measurements were transmitted by telephone to a central site. BP readings were recorded in a computerised report and then forwarded to patients and their physicians once a week. Physicians altered the dose or type of antihypertensive medication as needed by telephone or office visit. 61 patients were allocated to usual care.

Source of funding: Welch Allyn, Inc.

For correspondence: $\mathrm{Dr}$ M A Rogers, Joint Research Program for Pediatrics and Family Medicine, Medical College of Ohio, 1015 Garden Lake Parkway, Toledo, $\mathrm{OH} 43614$

USA. Fax +1419382 7876.

\section{Main outcome measures}

Mean arterial, systolic, and diastolic BP as measured by 24 hour ambulatory BP monitoring.

\section{Main results}

Analysis was by intention to treat. Difference in mean change in arterial, systolic, and diastolic BPs between the treatment and control groups was statistically significant (table).

\section{Conclusion}

In patients with essential hypertension, a home monitoring service improved mean arterial, systolic, and diastolic blood pressures better than usual care.

*See glossary.

\section{COMMENTARY}

Three quarters of patients with hypertension do not meet the definition of "controlled". The study by Rogers $e t$ al assesses the effect of home BP recording with an automated device combined with automated transmission of data by telephone to provide weekly reports for physicians and patients. Although the results are interesting and the conclusions reached are important, we must consider whether the process as described in the abstract was responsible for the results. Patients who were monitored at home had lower BP regardless of whether they had a change in medication. This finding raises the strong possibility that the $\mathrm{BP}$ effects seen were secondary to the attention to $\mathrm{BP}$ provided by intensive involvement in the protocol (checking BP 6 times $/ \mathrm{d}, 3 \mathrm{~d} / \mathrm{wk}$ ) or perhaps because of increased physician involvement or lifestyle changes. It is also reasonable to assume that the level of adherence to medications would be higher in this group of patients, and unfortunately medication compliance was not measured to resolve this issue. A more appropriate control group would have removed this source of bias by having participants in the study use the cuffs but send data only to the intervention group's physicians.

Determining the value of this service is important because at a cost of US $\$ 25$ per month many patients with hypertension will probably not be able to afford it. A consensus conference suggested that home BP monitoring might be better for long term management than (periodic) ambulatory $\mathrm{BP}$ monitoring ${ }^{2}$ and the cost would be less. Before this intervention is instituted, additional studies should be done that address the mechanism for $\mathrm{BP}$ improvement as well as the cost effectiveness of this approach.

Richard A Davidson, MD, MPH University of Florida College of Medicine Gainesville, Florida, USA

1 Joint National Committee on Detection, Evaluation, and Treatment of High Blood Pressure. Report of the Joint National Committee on detection, evaluation, and treatment of high blood pressure; 1997; NIH publication no. 98-4080: 3-6.

2 Herpin D, Pickering T, Stergiou G, et al. Consensus conference on self-blood pressure measurement. Clinical applications and diagnosis. Blood Press Monit 2000;5:131-5.

\section{Authors' response}

The monitoring service was designed to influence the behaviour of both physicians and patients. It was meant to provoke lifestyle changes. This was not bias but part of the intervention. Although separating the effects of physician and patient behaviours may be of academic interest, both ultimately affect blood pressure in everyday life.
Home monitoring service (HMS) v usual care for essential hypertension

\begin{tabular}{llll} 
Outcomes & HMS & Usual care & Mean difference $(95 \% \mathrm{Cl})$ \\
Arterial pressure $(\mathrm{mm} \mathrm{Hg})$ & -2.8 & +1.3 & $4.1(0.91$ to 7.38$)$ \\
\hline Systolic pressure $(\mathrm{mm} \mathrm{Hg})$ & -4.9 & -0.1 & $4.8(0.10$ to 9.37$)$ \\
\hline Diastolic pressure $(\mathrm{mm} \mathrm{Hg})$ & -2.0 & +2.1 & $4.1(0.93$ to 7.13$)$ \\
\hline
\end{tabular}

